\title{
Molecules and lons at Very Low Temperatures
}

\author{
Stefan Willitsch*
}

\begin{abstract}
The generation and study of 'cold' gas-phase molecules characterised by very low translational temperatures $T_{\text {trans }} \leq 1 \mathrm{~K}$ is an upcoming field of research in physical chemistry which has received considerable attention over the past years. A particular interesting form of cold molecules are ensembles of cold localised cations in ion traps which form ordered structures known as 'Coulomb crystals'. The present article reviews the experimental methods used for the generation of atomic and molecular Coulomb crystals and highlights recent experiments which take advantage of their intriguing properties in order to study chemical reactions at very low temperatures with single-particle sensitivity.
\end{abstract}

Keywords: Cold chemistry $\cdot$ Cold ions $\cdot$ Cold molecules $\cdot$ Laser cooling $\cdot$ Low-temperature reactions

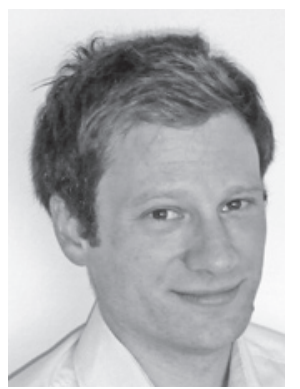

Stefan Willitsch graduated in natural sciences from ETH Zürich in 2000. He received his doctoral degree from ETH in 2004 for a thesis on the high-resolution photoelectron spectroscopy and photoionisation dynamics of reactive intermediates carried out under the supervision of Prof. Frédéric Merkt at the Laboratory for Physical Chemistry. From 2004-2007 he was a Junior Research Fellow at Christ Church College and the Physical and Theoretical Chemistry Laboratory at the University of Oxford (UK). $\mathrm{He}$ was appointed lecturer at University College London (UK) in 2007 and since 2008 he is an assistant professor at the Department of Chemistry at the University of Basel.
${ }^{\star}$ Correspondence: Prof. Dr. S. Willitsch Departement Chemie, Universität Basel Klingelbergstrasse 80

$\mathrm{CH}-4056$ Basel

E-Mail: stefan.willitsch@unibas.ch

\section{Introduction}

The study of 'cold' gas-phase atoms and molecules characterised by extremely low translational temperatures $\left(T_{\text {trans }}<1 \mathrm{~K}\right)$ represents one of the most fascinating fields of research in contemporary physics and chemistry. At very low temperatures the thermal de Broglie wavelength $\lambda_{\text {th }}$ of particles with mass $m$ given by

$$
\lambda_{\text {th }}=\frac{h}{\sqrt{2 \pi m k_{\mathrm{B}} T}}
$$

becomes larger than the extension of the particles themselves, e.g. $\lambda_{\text {th }}=11.5 \mathrm{~nm}$ for $\mathrm{Na}$ atoms at $T=1 \mathrm{mK}$. Consequently matter-wave effects strongly influence their physical and chemical properties, e.g. by leading to the emergence of unusual quantum phases like Bose-Einstein condensates or degenerate Fermi gases. ${ }^{[1]}$ The study of these phenomena has only become possible with the development of advanced cooling techniques, in particular laser cooling, which relies on the use of optical forces to slow down atoms in the gas phase. Since the first laser-cooling experiments in the late 1970s methods for the generation, trapping and manipulation of cold atomic gases down to temperatures of tens of $\mathrm{nK}$ have been established.[2] These achievements have been honoured with the recent award of two Nobel prizes in physics: 1997 for the development of laser cooling and 2001 for the generation of Bose-Einstein condensates from ultracold gases.

Because laser cooling is generally not applicable to molecules (see Section 2), the generation of translationally cold samples has until recently only been possible for atoms which excluded the study of a wealth of molecular processes at very low temperatures. Only over the past few years methods to produce molecular samples at translational temperatures in the sub-Kelvin range have been developed. These include Stark deceleration, Zeeman deceleration, buffer-gas cooling, collisional cooling, velocity selection, optical Stark deceleration, photoassociation and Feshbach association among others (see e.g. refs. [3-7] and references cited therein). The variety of recently developed cold-molecule sources reflects the interest in the many exciting potential applications such as ultra-high resolution spectroscopy, ${ }^{[8]}$ quantum-information processing ${ }^{[9]}$ and the study of chemical reactions in the cold regime.[6,10,11] Theoretical calculations predict that at ultralow collision energies many chemical reactions are dominated by quantum effects such as tunneling and the occurrence of reactive resonances which are sensitively dependent on the details of the potential energy surface of the relevant system. The study of such effects is not only of considerable interest in the context of low-energy collision physics, but also provides data for stringent tests of reactive-scattering models and high-level $a b$ initio calculations. ${ }^{[12,13]}$

Cold cations in ion traps represent a particularly interesting form of cold matter exhibiting unique properties which cannot be realised with cold neutrals. At temperatures of order tens of $\mathrm{mK}$ a trapped ion cloud undergoes a transition to an ordered structure also referred to as a 'Coulomb crystal' [14] Because of their strong localisation it is possible to observe and coherently manipulate single ions in small Coulomb crystals which thus represent attractive systems for the implementation of quantum-informationprocessing schemes. ${ }^{[15]}$ The ion trap situated in an ultrahigh-vacuum chamber constitutes a well-defined isolated environment in which interactions with the surroundings 
are minimised. These features in combination with the small Doppler line broadening prevalent at low temperatures make cold ions ideal systems for ultrahigh-resolution spectroscopic schemes of relevance for the development of new frequency standards and tests of fundamental physical concepts such as a possible time variation of natural constants. ${ }^{[16]}$ Finally, the possibility to observe single particles opens up intriguing perspectives for novel highly sensitive chemical studies at very low temperatures as detailed in Section 4. The present article reviews the generation and properties of cold ions in ion traps and highlights recent experiments from our laboratory in which their unusual properties have been used to study chemical reactions at very low temperatures with single-particle sensitivity.

\section{Ion Trapping and Cooling}

Over the past decades, trapping in radiofrequency (RF) ion traps has been established as a standard technique to store and manipulate atomic and molecular ions in mass spectrometry, ${ }^{[17]}$ charged-particle physics, ${ }^{[18]}$ laser cooling, ${ }^{[19]}$ and ion-trap based quantum-information processing. ${ }^{[15]}$ The most common type of ion-storage devices used in conjunction with laser-cooling experiments are linear Paul traps ${ }^{[20]}$ which typically consist of an assembly of four cylindrical electrodes arranged in a quadrupolar configuration as shown in Fig. 1 (a). Because charged particles cannot be trapped in three-dimensional space using static electric fields only, ${ }^{[17]}$ the ions are confined using a combination of RF and direct-current (DC) electric fields applied to the trap electrodes as indicated in Fig. 1 (a). The ions are confined along the central trap $(z)$ axis using DC potentials $V_{\text {end }}$ applied to the outer (endcap) electrodes. Confinement in the $(x, y)$ plane is achieved dynamically using rapidly oscillating RF potentials $V_{\mathrm{RF}}$ $=V_{\mathrm{RF}, 0} \mathrm{COS}\left(\Omega_{\mathrm{RF}} t\right)$ applied with opposite polarity to adjacent electrodes. $V_{\mathrm{RF} .0}$ and $\Omega_{\mathrm{RF}}$ denote the RF amplitude and frequency, respectively. The classical equations of motion of a single ion in the time-dependent electric field of the trap assume the form of Mathieu equations ${ }^{[17,21,22]}$

$$
\frac{d^{2} u}{d \tau^{2}}+\left[a_{u}+2 q_{u} \cos (2 \tau)\right] u=0
$$

with $u \in\{x, y, z\}$ and $\tau=(1 / 2) \Omega_{\mathrm{RF}} t$. The conditions for stable confinement of the ion depend on the values of the Mathieu parameters $a_{u}$ and $q_{u}$ which in turn depend on the RF and DC voltages applied to the trap electrodes, the RF frequency $\Omega_{\mathrm{RF}}$, the charge and mass of the ion and the geometry of the trap. ${ }^{[17,22]}$
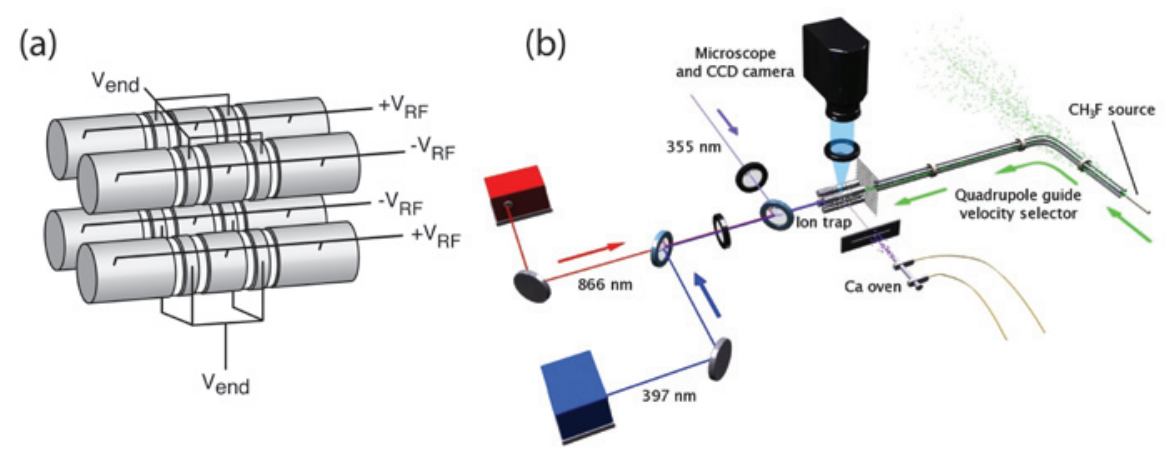

Fig. 1. (a) Schematic representation of a linear Paul trap. (b) Experimental setup to study ionmolecule reactions at very low translational temperatures $\left(T_{\text {trans }} \geq 1 \mathrm{~K}\right)$. Left-hand side: linear Paul trap used for the laser and sympathetic cooling of ions with cooling lasers and imaging system. Right-hand side: quadrupole-guide velocity selector to generate continuous beams of translationally cold polar neutral molecules. See text for details. Reproduced from ref. [10].

Laser cooling is performed by slowing down trapped atomic ions through momentum transfer from the repeated absorption of photons delivered by a laser beam. The laser frequency is slightly detuned to the red of the atomic resonance so that the mismatch is compensated by the Doppler shift in the frame of atoms moving towards the laser beam. In this way it is ensured that only atoms with a velocity component antiparallel to the propagation axis of the laser beam absorb photons and are thus decelerated. In a first approximation spontaneous re-emission of the photons does not lead to a net acceleration of the atoms because the emission is isotropic and the momenta imparted to the atom by the leaving photons cancel on average.

In contrast to neutral atoms in which a total of six laser beams directed along the positive and negative $(x, y, z)$ axes are required to achieve cooling in all three spatial dimensions, for ions it is sufficient to only cool along one direction because the trap ensures a coupling of all translational degrees of freedom. A setup for the laser cooling of ions is schematically depicted on the left-hand side of Fig. 1 (b). In our experiments $\mathrm{Ca}^{+}$ions are used which are laser cooled on the $4 \mathrm{~s}^{2} \mathrm{~S}_{1 / 2} \rightarrow 4 \mathrm{p}{ }^{2} \mathrm{P}_{1 / 2}$ transition at $397 \mathrm{~nm}$. The $4 \mathrm{p}^{2} \mathrm{P}_{1 / 2}$ state can also decay to the metastable $3 \mathrm{~d}^{2} \mathrm{D}_{3 / 2}$ state in which the atom is shelved so that the laser cooling is disrupted. To close the optical cycle another laser at $866 \mathrm{~nm}$ is used to repump population on the $3 \mathrm{~d}^{2} \mathrm{D}_{3 / 2} \rightarrow 4 \mathrm{p}^{2} \mathrm{P}_{1 / 2}$ transition.

In general the translational motion of molecules cannot be laser cooled because their complex energy level structure gives rise to a variety of decay channels which prevent the implementation of closed optical cooling cycles. Molecular ions, however, can be efficiently cooled when trapped in conjunction with a sample of laser-cooled atomic ions. ${ }^{[11,23-25]}$ In this case the Coulomb interaction between the ions ensures a rapid transfer of kinetic energy from the molecular to the atomic species from which it is removed by laser cooling. The efficiency of this 'sympathetic cooling' is limited by the magnitude of the momentum transfer between the laser-cooled atomic and the molecular ions which depends on the mass mismatch between the two species. Sympathetic cooling represents an extremely versatile technique to prepare a variety of translationally cold molecular ions ranging from diatomics ${ }^{[10,24]}$ and small to medium-sized polyatomics ${ }^{[25]}$ to multiply charged proteins. ${ }^{[26]}$

\section{Properties of Translationally Cold Ions in Ion Traps}

Fig. 2 shows false-colour fluorescence images of Coulomb crystals of laser-cooled $\mathrm{Ca}^{+}$ions taken in our laboratory. These images were obtained by monitoring the spontaneous emission produced during laser cooling using a charge-coupled-device (CCD) camera mounted on a microscope. Small ensembles of only a few ions usually

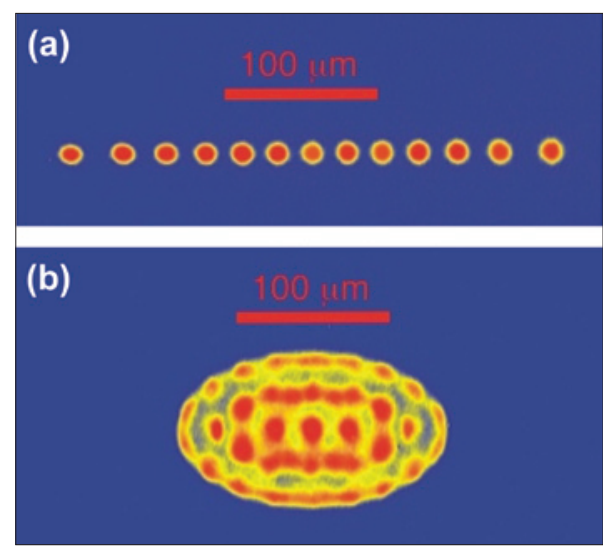

Fig. 2. False-colour fluorescence images of (a) a string of 13 laser-cooled $\mathrm{Ca}^{+}$ions and (b) a spheroidal Coulomb crystal of several tens of laser-cooled $\mathrm{Ca}^{+}$ions. 
form strings oriented along the central trap axis as shown in Fig. 2 (a). Every spot in the image represents a single localised laser-cooled $\mathrm{Ca}^{+}$ion. In larger samples with sizes exceeding tens of ions three-dimensional spheroidal structures are observed as a consequence of the symmetry of the trapping potential (see Fig. 2 (b)).

Ions in RF traps generally exhibit two types of motions which, under the conditions used in laser-cooling experiments, can be adiabatically separated:[21] a slow thermal ('secular') motion superimposed by a fast, oscillating 'micromotion' which is incited by the time-varying electric fields. During laser cooling only the secular motion of the ions is damped. The micromotion, however, is constantly driven by the RF fields and therefore not affected by laser cooling. As a consequence only ions situated on the central trap axis where the time-varying fields vanish for symmetry reasons are truly translationally cold whereas ions displaced from the trap axis exhibit a considerable amount of kinetic energy stored in the micromotion. The amplitude of an ion's micromotion depends on the magnitude of the local electric fields and therefore on its position in the trap. Consequently the average kinetic energy of the ions in a Coulomb crystal is strongly dependent on the trapping conditions and the size and shape of the ion ensemble. ${ }^{[11,27]}$ Large crystals are 'hotter' on average than small ones because a large number of ions is situated off axis in regions of increasing electric field strength towards the electrodes. This property can be used to tune the kinetic-energy distribution of the ion ensemble and thereby the collision energy in reactive-scattering experiments. ${ }^{[27]}$

\section{Chemical Reactions with Translationally Cold Ions}

One of the most intriguing applications of translationally cold molecules is the study of chemical reactions at extremely low temperatures. However, the realisation of low-energy reactive-collision experiments has until recently been prevented by the low number densities that can be produced with existing cold-molecule sources (typically on the order of $N=10^{8} \mathrm{~cm}^{-3}$ ). This can easily be seen by estimating the number of reactive collisions from the classical collision density $Z=\sigma v_{\text {rel }} N^{2}$. Using typical values of $v_{\text {rel }}=1000 \mathrm{~cm} \mathrm{~s}^{-1}$ for the relative velocity and $\sigma=10^{-15} \mathrm{~cm}^{2}$ for the reaction cross section, only on the order of 10 product molecules can be expected to be formed in a typical experimental volume of $10^{-3} \mathrm{~cm}^{-3}$ every experimental cycle. Obviously the detection of such a low number of product molecules represents a severe experimental challenge in the case of neu- trals. Using Coulomb crystals, however, it is possible to observe ion-molecule reactions with single localised ions and thus to study reactive processes with single-particle sensitivity even at extremely small rates on the order of a few reactive events per minute.

Fig. 1 (b) shows a schematic representation of a novel apparatus we have recently developed to study reactive collisions between translationally cold ions and neutral molecules. To our knowledge this experiment represents the first combination of two different cold-molecule sources in order to study reactive collisions at very low temperatures. Our new setup combines a facility for the laser and sympathetic cooling of ions in a linear Paul trap with a quadrupole-guide velocity selector for the generation of continuous beams of translationally cold polar molecules. $[10,11,27]$

The velocity selector serves to separate the slowest molecules from a thermal distribution in order to create samples of translationally cold molecules. The device used in our laboratory is an adaption of the design originally developed by Rangwala et al. ${ }^{[28]}$ consisting of four bent cylindrical electrodes arranged in a quadrupolar configuration as shown on the right-hand side of Fig. 1 (b). High voltages are applied with alternating polarities between adjacent rods in order to create a strong inhomogeneous electric field inside the assembly. A thermal ( $298 \mathrm{~K})$ beam of polar molecules is injected along the axis of the quadrupole. The electric field shifts the energy levels of the molecules (the Stark effect) and species in low-field-seeking Stark states, i.e. states whose energy increases with increasing field strength, experience a force directed towards the centre of the guide. When the molecules reach the bend of the velocity selector, only the slowest ones whose Stark energy exceeds their kinetic energy are forced around the corner whereas the fast molecules are lost from the guide and ultimately removed by pumping. With this simple method continuous beams of cold polar molecules with translational temperatures $T_{\text {trans }} \geq 1 \mathrm{~K}$ and fluxes of $10^{8}-$ $10^{9}$ molecules s ${ }^{-1}$ can be generated. ${ }^{[10,27]}$

The beam of translationally cold molecules is guided towards the centre of the ion trap containing an ion Coulomb crystal. Fig. 3 (a) shows the fluorescence image of a Coulomb crystal of laser-cooled $\mathrm{Ca}^{+}$ ions before exposure to the slow beam of neutrals. After bombarding the crystal with velocity-selected $\mathrm{CH}_{3} \mathrm{~F}$ molecules for about $30 \mathrm{~min}$, the image in Fig. 3 (b) is obtained. The $\mathrm{Ca}^{+}$ions react with $\mathrm{CH}_{3} \mathrm{~F}$ according to ${ }^{[29]}$

$$
\mathrm{Ca}^{+}+\mathrm{CH}_{3} \mathrm{~F} \rightarrow \mathrm{CaF}^{+}+\mathrm{CH}_{3}
$$

The $\mathrm{CaF}^{+}$product ions remain trapped under the present experimental conditions and are sympathetically cooled by the re- maining laser-cooled $\mathrm{Ca}^{+}$ions. Although the product ions cannot be observed directly, because they do not show laserinduced fluorescence at the frequency of the cooling laser, their presence manifests itself by the flattening of the central core of $\mathrm{Ca}^{+}$ions (see Fig. 3 (b)). The trapping and sympathetic cooling of the product ions is confirmed by mass spectrometry and the theoretical modelling of the observed fluorescence images using molecular dynamics (MD) simulations. ${ }^{[10,27]}$
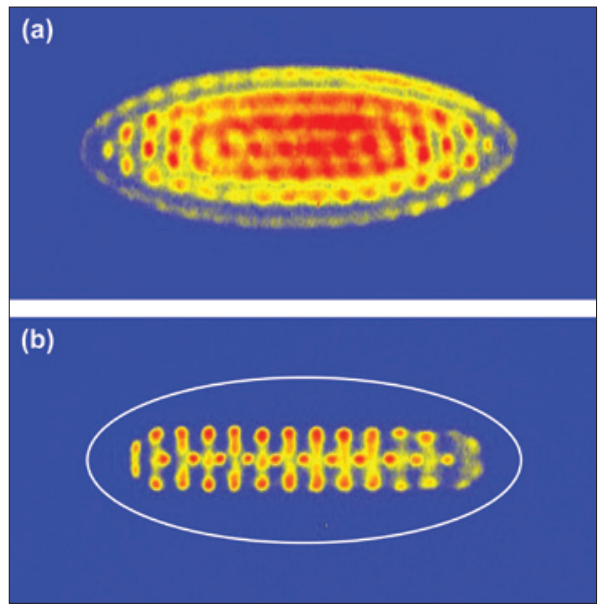

Fig. 3. False-colour fluorescence images of a $\mathrm{Ca}^{+}$Coulomb crystals before (a) and after (b) the reaction with velocity-selected $\mathrm{CH}_{3} \mathrm{~F}$ molecules. The white ellipse in (b) marks the boundaries of the original $\mathrm{Ca}^{+}$crystal shown in (a). The flattening of the central core of remaining $\mathrm{Ca}^{+}$ions in (b) is caused by the presence of sympathetically cooled $\mathrm{CaF}^{+}$ product ions, see text for details.

Fig. 4 (a) shows the decrease of the number of $\mathrm{Ca}^{+}$ions from a small Coulomb crystal as a function of the time of reaction with velocity-selected $\mathrm{CH}_{3} \mathrm{~F}$ molecules. The disappearance of single ions because of reactive collisions can clearly be observed illustrating that chemical reactions can be studied with single-particle sensitivity.

In larger crystals consisting of hundreds of ions the decrease of the $\mathrm{Ca}^{+}$ion number $N\left(\mathrm{Ca}^{+}\right)$follows a pseudo-first-order rate law of the form

$$
N\left(\mathrm{Ca}^{+}, t\right)=N\left(\mathrm{Ca}^{+}, t=0\right) \exp \left\{N\left(\mathrm{CH}_{3} \mathrm{~F}\right) k_{\mathrm{bi}} t\right\}
$$

From a fit of Eqn. (4) to the experimental data (solid line in Fig. 4 (b)), the secondorder rate constant $k_{\mathrm{bi}}$ was determined. At a voltage of $\pm 3.0 \mathrm{kV}$ applied to the electrodes of the velocity selector resulting in an average collision energy of $\left\langle E_{\text {coll }}\right\rangle / k_{\mathrm{B}} \approx 3$ $\mathrm{K}$, a value of $k_{\mathrm{bi}}=1.3(6) \times 10^{-9} \mathrm{~cm}^{3} \mathrm{~s}^{-1}$ was obtained. The rate constants obtained both at low and at room temperature $\left(k_{\mathrm{bi}}=4.2(4)\right.$ $\left.\times 10^{-10} \mathrm{~cm}^{3} \mathrm{~s}^{-1}\right)$ are considerably lower than 


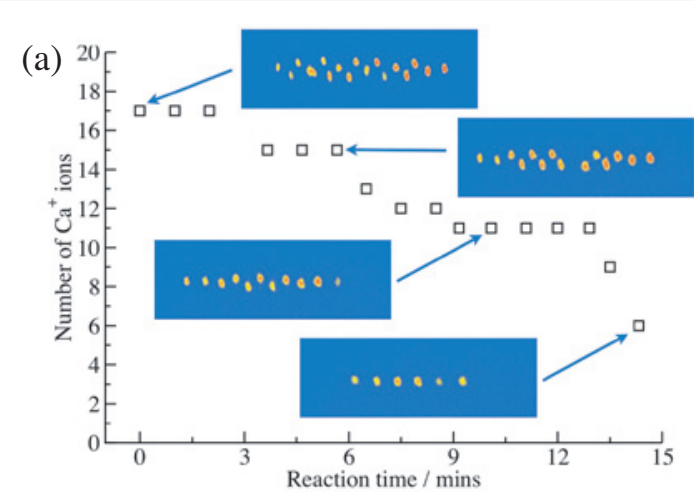

(b)

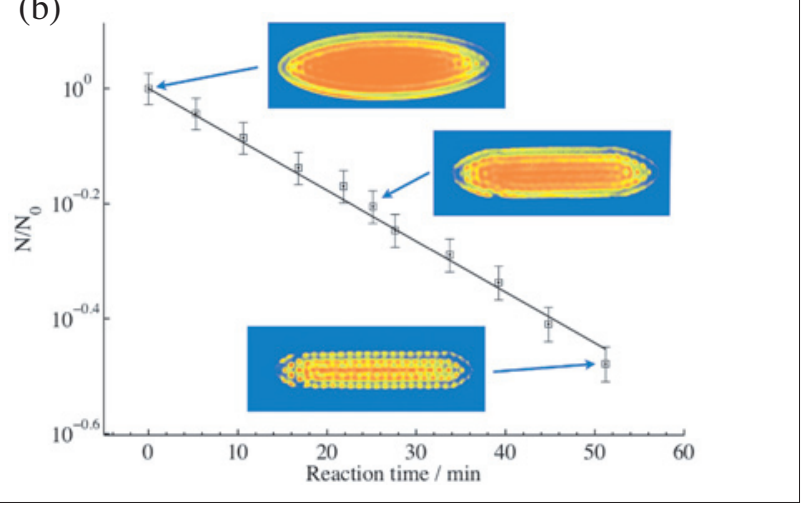

Fig. 4. Decrease of the number of laser-cooled $\mathrm{Ca}^{+}$ions as a function of the reaction time with velocity-selected $\mathrm{CH}_{3} \mathrm{~F}$ molecules for (a) a small Coulomb crystal of 17 ions and (b) a large Coulomb crystal of $\approx 250$ ions. The solid line in (b) represents a fit of the data to a pseudo-first order rate law from which the bimolecular rate constant $k_{\mathrm{bi}}$ of the reaction can be determined. Reproduced from ref. [11].

those predicted using quantum-mechanical capture models for ion-molecule reactive collisions ${ }^{[30]}$ indicating that the dynamics of this reaction cannot be described in terms of a simple ion-molecule capture process. ${ }^{[27]}$ The reduced rate constants are probably due to a submerged barrier along the reaction coordinate predicted by theoretical calculations which acts as a bottle neck for the reactive flux. ${ }^{[29,31]}$

With our new method it is not only possible to study reactions with laser-cooled ions, but more generally also with sympathetically-cooled species as has recently been demonstrated with the charge-exchange reaction $\mathrm{OCS}^{+}+\mathrm{ND}_{3} \rightarrow \mathrm{OCS}+\mathrm{ND}_{3}{ }^{+} .{ }^{[27]}$

\section{Conclusions and Outlook}

These results demonstrate the considerable potential for the application of cold ions in highly sensitive investigations of chemical reactions at low temperatures. The present experiment can be further developed in several directions to open up new fields of study. In the current setup neither the velocity-selected neutrals nor the sympathetically cooled molecular ions are quantum-state selected and usually exhibit a room-temperature Boltzmann distribution of internal state populations. ${ }^{[27]}$ Experiments with state-selected species, however, are essential to unravel the intricate details of the reaction dynamics at low collision energies and to study the dependence of the chemical reactivity on the quantum states of the reaction partners. ${ }^{[6]}$ State-selected cold molecular ions could be prepared by optical pumping of the population into a selected quantum state starting from translationally cold, but internally warm samples ${ }^{[32]}$ or by the sympathetic cooling of state-selectively produced molecular ions. ${ }^{[11,33]}$ Samples of state-selected neutral molecules which are equally required for the realisation of fully state-selected ion-molecule collision experiments can be produced using alternative cold-molecule sources such as a Stark decelerator. ${ }^{[4]}$ Using Stark deceleration of pulsed molecular beams has the additional advantage of producing colder samples compared to velocity selection (down to translational temperatures of tens of $\mathrm{mK}$ after trapping[34]). Even lower collision energies corresponding to $\mathrm{mK}$ could be reached in experiments which combine Coulomb crystals with trapped ultracold atomic gases prepared, e.g. by laser cooling in a magnetooptical trap. Developments in these directions are under way in our laboratory.

\section{Acknowledgements}

The experiments reviewed in this article were developed in collaboration with Prof. Timothy P. Softley, University of Oxford, UK. This project formed part of the $\mathrm{PhD}$ theses of Martin Bell, Alexander D. Gingell and James Oldham whose efforts and contributions are especially acknowledged. Our current work on stateselected molecular Coulomb crystals is funded by the Swiss National Science Foundation under grant no. PP002-118921.

Received: January 9, 2009

[1] C. J. Pethick, H. Smith, 'Bose-Einstein Condensation in Dilute Gases', Cambridge University Press, 2001.
[2] H. J. Metcalf, P. van der Straten, 'Laser Cooling and Trapping', Springer, New York, 1999.

[3] 'Interactions in Ultracold Gases: From Atoms to Molecules', Eds. M. Weidemüller, C. Zimmermann, Wiley-VCH, Weinheim, 2003.

[4] H. L. Bethlem, G. Meijer, Int. Rev. Phys. Chem. 2003, 22, 73 .

[5] J. Doyle, B. Friedrich, R. V. Krems, F. MasnouSeeuws, Eur. Phys. J. D 2004, 31, 149.

[6] R. V. Krems, Phys. Chem. Chem. Phys. 2008, 10, 4079.

[7] J. M. Hutson, P. Soldán, Int. Rev. Phys. Chem. 2006, 25, 497.

[8] T. Zelevinsky, S. Blatt, M. M. Boyd, G. K Campbell, A. D. Ludlow, J. Ye, ChemPhysChem 2008, 9, 375 .

[9] D. DeMille, Phys. Rev. Lett. 2002, 88, 067901.

[10] S. Willitsch, M. Bell, A. Gingell, S. R. Procter, T. P. Softley, Phys. Rev. Lett. 2008, 100, 043203.

[11] S. Willitsch, M. T. Bell, A. Gingell, T. P. Softley, Phys. Chem. Chem. Phys. 2008, 10, 7200.

[12] P. F. Weck, N. Balakrishnan, Int. Rev. Phys. Chem. 2006, $25,283$.

[13] J. M. Hutson, P. Soldán, Int. Rev. Phys. Chem. 2007, $26,1$.

[14] The term 'colulomb crystal' is conventionally used in analogy to ordered structures of electrons in solids originally predicted by E. Wigner, Phys. Rev. A 1934, 46, 1002. Strictly speaking, cold ions in ion traps do not form crystalline structures owing to the lack of periodicity of the trap potential.

[15] R. Blatt, D. Wineland, Nature 2008, 453, 1008.

[16] T. Rosenband, D. B. Hume, P. O. Schmidt, C. W. Chou, A. Brusch, L. Lorini, W. H. Oskay, R. E. Drullinger, T. M. Fortier, J. E. Stalnaker, S. A. Diddams, W. C. Swann, N. R. Newbury, W. M Itano, D. J. Wineland, J. C. Bergquist, Science 2008, 319, 1808.

[17] R. E. March, J. F. Todd, 'Quadrupole Ion Trap Mass Spectrometry', John Wiley \& Sons, 2nd edition, 2005.

[18] 'Trapped Charged particles and Fundamental Interactions', Eds. K. Blaum, F. Herfurth, N. 749 in Lecture Notes in Physics, Springer Verlag, 2008.

[19] D. J. Berkeland, J. D. Miller, J. C. Bergquist, W. M. Itano, D. J. Wineland, J. Appl. Phys. 1998 83, 5025.

[20] J. D. Prestage, G. J. Dick, L. Maleki, J. Appl. Phys. 1989, 66, 1013 .

[21] D. Gerlich, Adv. Chem. Phys. 1992, 82, 1.

[22] M. Drewsen, A. Brøner, Phys. Rev. A 2000, 62, 045401.

[23] T. Baba, I. Waki, Jpn. J. Appl. Phys. 1996, 35, 1134.

[24] K. Molhave, M. Drewsen, Phys. Rev. A 2000, 62, 011401.

[25] A. Ostendorf, C. B. Zhang, M. A. Wilson, D. Offenberg, B. Roth, S. Schiller, Phys. Rev. Lett. 2006, 97, 243005.

[26] D. Offenberg, C. B. Zhang, Ch. Wellers, B. Roth, S. Schiller, Phys. Rev. A 2008, 78, 061401.

[27] M. Bell, A. Gingell, J. Oldham, T. P. Softley, S. Willitsch, Faraday Discuss. 2009, 142, accepted for publication.

[28] S. A. Rangwala, T. Junglen, T. Rieger, P. W. H. Pinkse, G. Rempe, Phys. Rev. A 2003, 67, 043406 .

[29] J. N. Harvey, D. Schröder, W. Koch, D. Danovich, S. Shaik, H. Schwarz, Chem. Phys. Lett. 1997, 273,164

[30] D. C. Clary, Chem. Phys. Lett. 1995, 232, 267.

[31] H. Sabbah, L. Biennier, I. R. Sims, Y. Georgievskii, S. J. Klippenstein, I. W. M. Smith, Science 2007, 317,102 .

[32] I. S. Vogelius, L. B. Madsen, M. Drewsen, Phys. Rev. Lett. 2002, 89, 173003.

[33] S. Willitsch, F. Merkt, Int. J. Mass Spectrom. $\mathbf{2 0 0 5}, 245,14$.

[34] S. Y. T. van de Meerakker, N. Vanhaecke, M. P. J. van der Loo, G. C. Groenenboom, G. Meijer Phys. Rev. Lett. 2005, 95, 013003. 